\title{
Buenas prácticas en educación inclusiva
}

\section{Good practices in inclusive education}

\author{
Joan Jordi Muntaner Guasp ${ }^{1}$ \\ joanjordi.muntaner@uib.es \\ María Rosa Rosselló Ramón \\ mrosa.rossello@uib.es \\ Begoña de la IGlesia Mayol \\ bego.delaiglesia@uib.es \\ Universidad de les Illes Balears, España
}

\section{Resumen:}

En estos últimos años, han ido apareciendo una amplia variedad y cantidad de experiencias de aula que han implementado con éxito prácticas inclusivas, así como centros escolares que han iniciado su andadura hacia la inclusión educativa. Sin embargo, todavía no existe un consenso pleno sobre el tema $y$, en muchos casos, se producen prácticas que esconden experiencias excluyentes y contradictorias.

En este contexto, el artículo parte de la necesidad de revisar qué caracteriza a las buenas prácticas inclusivas y qué actuaciones deben impulsarse para generar los cambios necesarios para consolidar una escuela inclusiva. En respuesta a tales interrogantes se analizan las principales aportaciones, a la vez que se recogen los cambios sustanciales que deben impulsarse en la cultura de los centros y las aulas.

\section{Palabras clave:}

Inclusión educativa; buenas prácticas inclusivas; profesorado; cambio educativo.

\begin{abstract}
:
Over the last few years a wide variety and quantity of classroom experiences of an inclusive nature have appeared with great success. The same can be said of schools who have started the move towards inclusive education. Nevertheless, there is still no common consensus on the matter and, in many cases, there are practices underlaid by discriminatory and contradictory experiences. In this context, this article is founded on the need to review exactly what characterizes good inclusive practices and what actions should be pushed ahead to generate the changes needed to consolidate inclusive schooling. In response to these questions, this article discusses the main contributions in the field while, at the same time, it highlights the substantial changes that need to be pursued in school and classroom culture.
\end{abstract}

\section{Key words:}

Inclusive education; good inclusive practices; teachers; educational change.

\section{Dirección para correspondencia (correspondence address):}

Joan Jordi Muntaner Guasp. Dpto. de Pedagogía Aplicada y Psicología de la Educación. Facultad de Educación. Educación. Universitat de les Illes Balears. Edifici Guillem Cifre de Colonya. Campus Universitari. Carretera de Valldemossa, Km. 7,5. 07122 Palma de Mallorca (España). 


\section{Résumé:}

Au cours de ces dernières années, un grand nombre d'expériences diverses ont vu le jour concernant des classes ayant appliqué avec succès des pratiques inclusives ainsi que des établissements s'étant engagés dans la direction de l'inclusion scolaire. Cependant, il $n^{\prime} y$ a pas encore de consensus sur cette question et il s'avère que, dans de nombreux cas, derrière certaines pratiques se cachent des expériences exclusives et contradictoires. Dans ce contexte, cet article naît de la nécessité de passer en revue ce qui caractérise les bonnes pratiques inclusives et de déterminer les actions devant être encouragées en vue de générer les changements nécessaires à la consolidation d'une école inclusive. Afin de répondre à ces questions, nous analyserons les principaux apports puis nous exposerons les changements substantiels devant être introduits dans la culture des établissements et des salles de classe.

\section{Mots clés:}

Inclusion scolaire; bonnes pratiques inclusives; personnel enseignant; changement éducatif.

Fecha de recepción: 27-5-2014

Fecha de aceptación: 19-9-2014

\section{Introducción}

Desde que en 1990 en la Declaración Mundial sobre Educación para Todos comenzó a acuñarse el modelo de educación inclusiva hasta la actualidad, mucho han cambiado las cosas y grandes son los avances que han propiciado un desarrollo y un asentamiento del modelo en los foros nacionales e internacionales. Autores como Arnaiz (2011, 2012), Ainscow (2005a, 2005b, 2012), Booth (2006), Booth y Ainscow (2002), Echeita (2006), Casanova (2011), López Melero (2004), Giné (2010), Florian y Black-Hawkins (2010), Verdugo y Parrilla (2009), entre otros, han elaborado un amplio y relevante cuerpo de conocimientos sobre educación inclusiva, aportando un bagaje potente y significativo. Si bien, como afirman Echeita y Ainscow (2011) todavía existe una cierta confusión, y el término se conceptualiza de múltiples maneras (Ainscow y Miles, 2008). En España, por ejemplo, abundan las publicaciones que contrastan los conceptos de Integración e Inclusión, con el objetivo de potenciar una perspectiva inclusiva y romper con las ideas y valores que sustentan un modelo educativo de corte tradicional, alejado de las necesidades que plantea la sociedad del siglo XXI.

Esta confluencia de propuestas, de investigaciones, de trabajos y experiencias ha proporcionado avances importantes en el desarrollo y aplicación de la educación inclusiva. Sin embrago, debemos reconocer que 
nos falta mucho camino por recorrer y que necesitamos no desfallecer y sentirnos fuertes en las ideas y valores ante la falta de consenso, de arraigo y de expansión de este modelo educativo en nuestros centros y aulas escolares. Esta situación plantea la necesidad de dar un nuevo impulso a este proceso y para ello nos hacemos una serie de preguntas, que serán el eje conductor de este artículo:

1. ¿Cómo podemos y debemos continuar?

2. ¿Cómo debemos consolidar las prácticas inclusivas ya iniciadas e implantadas?

\section{Equidad y calidad, claves de la educación inclusiva}

La educación inclusiva es un cambio global del sistema educativo, que afecta a todo el alumnado con un doble objetivo: conseguir el éxito de todos, sin excepciones, en la escuela; y luchar contra cualquier causa o razón de exclusión, en cualquiera de sus variantes de segregación y/o discriminación.

La Agencia Europea para el Desarrollo de la Educación del Alumnado con Necesidades Educativas Especiales (2009) señala que para el progreso de la educación inclusiva se necesita la presencia de tres dimensiones básicas: calidad, equidad e inclusión; siendo la calidad y la equidad cruciales para garantizar la educación inclusiva y entendiendo, además, que la inclusión y la calidad son variables recíprocas en el modelo educativo actual.

De hecho, la educación de calidad implica un doble equilibrio entre:

a) Ofrecer una educación común para todo el alumnado y, al mismo tiempo, reconocer y ajustarse a las necesidades de aprendizaje y características individuales de cada uno de los alumnos.

b) $\mathrm{Y}$ entre la excelencia y la equidad, en el sentido en que no se puede hablar de calidad cuando tan sólo una minoría de alumnos aprende lo necesario para participar en la sociedad y desarrollar su proyecto de vida (Blanco, 2008)

De algún modo, cuanta más coherencia se da entre cada una de las parejas de estas variables, mayor calidad tiene la propuesta educativa, y viceversa, a medida que se altera este equilibrio, en una u otra dirección, la propuesta pierde calidad. 
Para alcanzar tales dimensiones es importante, como afirma Blanco $(2008,10)$, tener presente la equidad, lo que supone proporcionar a cada persona las ayudas y los recursos que necesita para que esté en igualdad de condiciones de aprovechar las oportunidades educativas y de aprender a niveles de excelencia. Por su parte, la OCDE (2012) señala que la equidad en educación significa que las circunstancias personales o sociales, como género, origen étnico o antecedentes familiares, no obstaculicen el logro del potencial educativo y que todos los individuos alcancen al menos un nivel mínimo de habilidades.

La educación inclusiva persigue ofrecer una respuesta de calidad a la diversidad existente en las aulas, desde un planteamiento equitativo, no basado en enfoques asistenciales, compensatorios o focalizados, sino con un fuerte carácter preventivo y global. Este es el gran desafío al que se enfrentan las escuelas y los docentes en la actualidad, lo que siguiendo a Blanco "exige cambios sustantivos en las concepciones, actitudes, el currículo, las prácticas pedagógicas, la formación de los docentes, los sistemas de evaluación y la organización de las escuelas" (Blanco, 2008, 8)

En la consolidación del modelo de educación inclusiva es necesario aunar posiciones, delimitar claramente el concepto y construir referentes válidos y potentes que iluminen el camino. En este sentido, es relevante la propuesta realizada por Ainscow, Booth y Dyson (2006), al definir la inclusión educativa como la presencia, la participación y el progreso de todos los alumnos en el aula y el centro ordinario:

- Presencia: En el camino hacia la inclusión, la primera condición es que todos los alumnos estén siempre presentes en todas las actividades, situaciones de aprendizaje y experiencias que se desarrollen con el grupo de referencia.

- Participación: La segunda condición es que todos los alumnos, sin ninguna excepción, participen en todas las actividades, situaciones de aprendizaje y experiencias planificadas para el grupo de referencia. Ello no significa que todos los alumnos deban participar de la misma manera, sino que debe ofrecerse un abanico suficientemente amplio de posibilidades, que permita esta participación a todos y cada uno de los alumnos respetando sus capacidades y potencialidades.

- Progreso: El desarrollo de un proceso educativo requiere necesariamente que todos los alumnos en todas las experiencias, activi- 
dades y situaciones planteadas alcancen un aprendizaje óptimo, aunque no sea necesariamente el mismo para todos y cada uno de los alumnos.

\section{De la implementación a la consolidación de las prácticas inclusivas}

Los cambios y las innovaciones en educación pasan por tres grandes fases: la iniciación, la implementación y la consolidación. Para alcanzar esta última fase se requiere una situación de choque entre los procedimientos y estrategias tradicionales y las nuevas formas de entender $y$ realizar las prácticas educativas, que acaban imponiéndose por la determinación y la convicción de los profesionales que las aplican.

La educación inclusiva, al igual que cualquier otro modelo educativo, tiene en los valores y las creencias los fundamentos que la sostienen y se explican por una teoría, es decir, por unos referentes que justifican las decisiones y las prácticas realizadas. Conocer cuáles son estos referentes que determinan la perspectiva de análisis e interpretación de la realidad educativa actual, nos proporciona los fundamentos científicos y reales de nuestra propuesta.

De manera general, se dan dos posturas antagónicas en el modo de tratar la diversidad existente en cualquier grupo humano, posturas que se observan también en los centros escolares actuales. Por una parte, los principios, valores y creencias que se fundamentan en la lógica de la homogeneidad. Esta perspectiva busca composiciones uniformes y categoriza las diferencias, distinguiendo entre alumnos normales y alumnos diversos, alumnos de necesidades educativas especiales, alumnos inmigrantes, etc. La aplicación de esta perspectiva conduce a un modelo de escuela selectiva, que busca reducir la diversidad a partir de programas específicos dirigidos a colectivos concretos, que conducen a la segregación y discriminación, con el pretexto que, con ello, se beneficia a los miembros de estos colectivos.

Frente a esta opción, se encuentra la lógica de la heterogeneidad. Desde esta perspectiva, se reconoce y acepta la diversidad de todas las personas sin pretender igualarlas ni cambiar sus características, centrando la atención en modificar el contexto y en desarrollar estrategias que respondan adecuadamente a esta diversidad. Puesto que todos los alum- 
nos son diversos, hay que potenciar el aprendizaje de todos desde la pertenencia al grupo y al respeto de las características individuales. Esta posición conduce a un modelo de escuela inclusiva, que se caracteriza por atender a todos los alumnos y por responder a la diversidad real y natural de cualquier grupo humano.

Entre estas dos posiciones, se ha creado una falsa postura intermedia, a la que denominamos postura de camuflaje. Desde esta perspectiva se asumen los principios de la lógica de la heterogeneidad pero se combinan con prácticas discriminatorias, selectivas y categorizadoras. El peso de la tradición, las dudas, la falta de convicción, la presión de antiguas ideas y valores... mezclan tales prácticas con posiciones y planteamientos inclusivos. Estas experiencias son las que impiden una verdadera consolidación de prácticas inclusivas en nuestros centros y aulas ordinarias, puesto que al no ser tales, impiden el desarrollo y la extensión de verdaderas prácticas inclusivas.

La consolidación de prácticas inclusivas pasa ineludiblemente por determinar y realizar iniciativas y experiencias positivas de innovación y mejora, de modo que se den los siguientes indicadores:

- Entender la diversidad como un hecho natural entre las personas de cualquier grupo sin establecer categorías, puesto que al hacer ésto se añade una variable de valor, donde unos ganan y otros pierden. La práctica inclusiva acepta la realidad y las características de todos sus alumnos desde una mirada positiva.

- El reconocimiento explícito de la riqueza inherente a la diversidad, conduce a plantear el objetivo de una actuación educativa centrada no en la persona y sus características, sino en las condiciones y oportunidades que se le ofrecen. Nuestra actuación tiene como objetivo modificar el entorno para que todos puedan aprender al máximo de sus posibilidades, valorando sus aprendizajes y trabajando desde la aceptación incondicional de las capacidades de todos los alumnos.

- Las prácticas inclusivas se fundamentan en la aplicación de agrupamientos heterogéneos, donde se refleja la diversidad real presente en todos los grupos y la incorporación natural de los apoyos necesarios para garantizar la igualdad de oportunidades de todos los alumnos sin excepciones.

- Por último, las prácticas inclusivas cumplen tres condiciones clave: garantizan la presencia, la participación y el aprendizaje de 
todos los alumnos en todas las propuestas que se hacen en el aula y en la escuela.

Para ello es imprescindible la convicción de toda la comunidad educativa, con especial atención al profesorado puesto que es la piedra angular en todo este proceso. Por eso es necesario incorporar en la cultura de los centros la búsqueda de mejoras como una práctica cotidiana y común en la escuela, no como un hecho puntual u ocasional. En esta misma línea inciden Murillo y Krichesky $(2012,41)$ :

"Es necesario insistir entonces en la importancia de que los centros educativos se conviertan en espacios que fomenten no sólo el desarrollo integral de sus alumnos sino también, y como medio para ello, el desarrollo profesional de sus docentes".

Progresivamente, debe contarse también con la participación e implicación de familias, administración, personal no docente y el conjunto de la sociedad.

En estos últimos años, han ido apareciendo una amplia variedad y cantidad de experiencias que han implementado con éxito prácticas inclusivas, así como centros escolares que han iniciado su andadura hacia la inclusión educativa. Podemos encontrar en la bibliografía nacional distintas y variadas experiencias de buenas prácticas, de todas ellas destacar las comunidades de aprendizaje, propuestas por el grupo CREA de la Universidad de Barcelona (Elboj, Puigdellivol, Soler y Valls, 2002) donde se plantean las directrices y los procesos a seguir para la transformación de los centros escolares. Ejemplos de experiencias en este sentido se describen en Martínez y Gómez (2013). Otro instrumento clave para favorecer y potenciar las buenas prácticas inclusivas es el Index for Inclusion (Booth y Ainscow, 2002); una experiencia de su aplicación la encontramos en el CEIP Els Xiprers (Agell, Sala y Torrent, 2009). Al mismo tiempo se dan prácticas que se contemplan y se presentan como inclusivas, pero realmente siguen siendo segregadoras y discriminatorias; el ejemplo más claro es el de los agrupamientos flexibles (Albericio, 2001). Por todo ello, como indica Ainscow (2005a), en estos momentos es necesario un nuevo impulso, capaz de determinar qué debe hacerse para afianzar el progreso alcanzado hasta la fecha y generalizar buenas prácticas que permitan consolidar este modelo educativo. 
El término buenas prácticas no es fácil de acotar, pues no existe unanimidad en su definición. Sin embargo, sabemos que tiene su origen en el mundo empresarial y hace referencia a algo que funciona y que ha obtenido los resultados esperados. La UNESCO en el marco de su programa Management of Social Transformations (MOST) Programme (Santiso, 2002), ha especificado cuáles son los atributos de una buena práctica educativa y los rasgos que la caracterizan. En términos generales, las buenas prácticas han de ser:

- Innovadoras, desarrollan soluciones nuevas o creativas.

- Efectivas, demuestran un impacto positivo y tangible sobre la mejora.

- Sostenibles, por sus exigencias sociales, económicas y medioambientales, pueden mantenerse en el tiempo y producir efectos duraderos

- Replicables, sirven como modelo para desarrollar políticas, iniciativas y actuaciones en otros lugares.

Trabajar con buenas prácticas, tal como señala Escudero (2009), supone identificar, diseminar y compartir conocimientos y experiencias exitosas y contrastadas en el logro de objetivos, lo que en el caso de la educación inclusiva implica:

a) Aplicar prácticas de tercera generación (Wehmeyer, 2009, 59), lo que supone poner el énfasis en la calidad del programa educativo del aula. Sin desdeñar lo que representaron las prácticas de primera o segunda generación, en estos momentos se persigue asegurar programas de alta calidad educativa para todos los alumnos y alcanzar altas cotas de éxito y de participación de todos en el aula de educación general.

b) Determinar los objetivos a lograr a partir de la identificación y superación de las barreras a la participación y al aprendizaje, como explica Ainscow (2005a), recogiendo evidencias que muestren si los alumnos -particularmente aquellos con mayor riesgo de marginación o exclusión- realmente han participado en todas las actividades y experiencias planteadas en el aula ordinaria y, consecuentemente, si han aprendido.

Todo ello requiere la implicación y el compromiso del profesorado, tal como explica Escudero $(2009,128)$ : 
...es improbable pensar en buenas prácticas docentes donde no haya existido una preparación pertinente (en valores, creencias, concepciones, metodologías, etc) donde no se haya podido acceder a buenos materiales y experiencias de otros, o donde no haya sido posible constituir equipos docentes.

De este modo, las buenas prácticas inclusivas son aquellas que proporcionan programas de alta calidad educativa para todos sin excepciones, y, además, se centran en la presencia, participación y logros de todos los alumnos. Conocer y describir estas buenas prácticas puede ayudar a identificar los factores que influyen directamente en promocionar avances inclusivos en el sistema educativo, avances que es posible clasificar en dos bloques:

- Cambios en el sistema educativo

- El desarrollo de escuelas inclusivas

\subsection{Cambios en el sistema educativo}

La inclusión en España aparece como continuación del programa de integración, heredando las limitaciones, confusiones y desvíos que protagonizó la incorporación de los alumnos con discapacidad en las escuelas ordinarias (Muntaner, 2010).

La manera de entender y tratar la diversidad del alumnado desde una perspectiva inclusiva exige cuestionar el orden vigente, puesto que cuando no ocurren transformaciones culturales, tampoco las prácticas cambian sustancialmente, sino que simplemente se adaptan. En palabras de Escudero y Martínez $(2011,98)$ :

La integración llevó consigo recursos materiales y humanos como habilitación de espacios, eliminación de barreras físicas, creación de nuevos roles especializados (profesores de pedagogía terapéutica; logopedas, compensatoria, servicios y profesionales externos especializados). Hubo una suma de elementos yuxtapuestos, no cambios sustantivos en las regularidades culturales y prácticas preexistentes.

La inclusión exige reformar las estructuras generales, la pedagogía y los procedimientos de trabajo de la educación básica, pues se trata de un proceso permanente de eliminación de obstáculos a la asistencia escolar y al aprendizaje, tanto en la escuela como en la sociedad. Como 
escriben Halinen y Jarvinen (2008) nuestro principal reto consiste en aprender a convivir con la diversidad y el multiculturalismo recientes de la sociedad y la escuela.

El objetivo es ahora ayudarnos a identificar aquellos factores que influyen directamente en proponer avances inclusivos dentro del sistema educativo vigente y plantear los cambios necesarios, de manera que sea factible avanzar en el camino de la inclusión. Halinen y Jarvinen (2008, 122-123) proponen cinco esferas de desarrollo:

1. Para asegurar una educación básica igual para todos es preciso contraer el compromiso mental y económico de pensar que todas las personas tienen derechos y responsabilidades como miembros de la sociedad y que son capaces de contribuir a ella.

2. Para que la inclusión cumpla su cometido, todos los niños deben asistir a la escuela general y seguir trabajando para que todos terminen, sin antes abandonar, al menos la educación básica.

3. La inclusión exige voluntad colectiva y una cultura de trabajo común en la que se valora la participación de todos los miembros de la sociedad. Debemos contar con modelos de trabajo basados en la colaboración, donde todos puedan contribuir equitativamente, contando con el respaldo de las familias. Cuando esto ocurre, la diversidad se percibe como una virtud y un recurso.

4. La inclusión depende en gran medida de los métodos positivos aplicados por los maestros y la sólida capacidad profesional de éstos. Los maestros necesitan una formación inicial de alta calidad y oportunidades para poder continuar su desarrollo profesional mediante la capacitación en el empleo y el mantenimiento de contactos profesionales con otros maestros.

5. El currículo debe ser la expresión de los valores básicos de la inclusión en la educación y de la voluntad consensuada de desarrollar esta educación.

Si la visión finlandesa aporta una perspectiva social de cual es el camino a seguir para cambiar el sistema educativo hacia un modelo inclusivo, desde una posición más pedagógica Escudero y Martínez (2012) apuntan la necesidad de superar la tentación más pragmática, rápida y menos comprometida de seguir poniendo en marcha numerosos medidas parciales y marginales y, en su lugar, reclamar sinergias múltiples en diversos niveles para dar respuesta a la diversidad de forma inclusiva. 
Algunas propuestas para hacer cambios profundos en el sistema y en la cultura escolar, desde un planteamiento inclusivo, pueden ser, entre otras, las que recogen Escudero y Martínez (2012):

a) El sistema escolar debe asumir la prioridad de que todos los alumnos aprendan al máximo de sus posibilidades.

b) Se debe trabajar para reducir las desigualdades de oportunidades, así como cambiar las desigualdades presentes en los contenidos y resultados.

c) Apostar por el éxito en los estudios es algo que lleva inexorablemente a interpelar y transformar el orden escolar vigente.

d) Reclamar centros, currículos y aulas habitables para todos. No con contenidos devaluados o rebajados, sino rigurosos, relevantes, significativos, conectados con el mundo de los alumnos y aptos para enriquecer sus capacidades de resolver problemas, de comunicar, de ser creativos, de establecer vínculos con los demás y de lograr una imagen positiva de sí mismos.

e) Hacer efectivo que los lugares de enseñanza sean también lugares de formación docente en los que aprender, innovar, observar y analizar lo que va sucediendo y sostener un pulso reflexivo y crítico de mejora, como caminos necesarios por los que transitar.

f) Al igual que la formación inicial, la selección, el desarrollo y los aprendizajes del profesorado son necesarios para avanzar en experiencias y resultados escolares equitativos. Los servicios y profesionales que trabajan en la administración tienen que aprender y formarse para cumplir sus cometidos, crear condiciones favorables para que centros y docentes desarrollen capacidades y compromisos con la mejora.

g) Tener una visión de los centros escolares como espacios integrados de servicio donde se amplíen las oportunidades de formación, abiertas a fomentar las alianzas entre los centros, las familias, la comunidad, otros servicios y las empresas.

Estas propuestas pueden ayudar a provocar cambios en los planteamientos generales del sistema educativo, que permitirán eliminar barreras y potenciarán los facilitadores necesarios en el avance de la educación inclusiva, proyectándose en la cultura y la vida cotidiana de los centros escolares. 


\subsection{Desarrollo en la escuela}

La educación inclusiva es una cuestión de derechos, donde todos los alumnos de la comunidad aprenden juntos, independientemente de sus condiciones personales, sociales o culturales. Se trata de lograr una escuela que se adapte a las características de los alumnos y no a la inversa. Por todo ello, la inclusión -afirman Marchesi y otros $(2009,4)-$ tiene que ver con un proceso de búsqueda que cada comunidad escolar debe llevar a cabo, para encontrar mejores formas y más eficaces de responder a la diversidad del alumnado. Se trata, pues, de un proceso de mejora y de desarrollo institucional en el que se compromete a toda la comunidad escolar.

La realización de este proceso en cada centro y contexto determinado precisa la intervención de un liderazgo decidido, basado en las creencias y valores como la equidad y la justicia social, que desde una postura democrática y compartida, favorezca la transformación cultural y en consecuencia los cambios en las prácticas docentes que permitan el desarrollo de procesos inclusivos. Como afirman García y otros (2012, 262):

Los centros inclusivos diseñan soluciones novedosas centradas en producir cambios significativos y duraderos en la organización, en la enseñanza o en las interacciones personales para adecuarse a las nuevas demandas de la realidad escolar, en ellas se observan mejores resultados en la adquisición de aprendizajes funciones y habilidades sociales

El liderazgo en los centros escolares juega un importante papel para afrontar los retos que plantea la diversidad y remover las barreras que dificultan la inclusión. Ainscow y Miles (2008) concluyen que los líderes escolares deben atender a tres grandes tipos de tareas:

- Impulsar nuevos significados sobre la diversidad.

- Promover prácticas inclusivas en las escuelas.

- Establecer vinculaciones entre las escuelas y las comunidades.

Para desarrollar la educación inclusiva en un centro escolar, el liderazgo desempeña una labor fundamental en la consolidación de este modelo educativo. González (2008) recoge los rasgos principales que deben darse: 
1. Se plantea un liderazgo democrático y participativo. Se requiere el compromiso y el trabajo conjunto de reflexión, diálogo, indagación y acción, que implica a todos los miembros del centro.

2. Desplegar un liderazgo alrededor de un propósito común, fundamentado en valores y principios de inclusión.

3. Estos principios y valores deben reflejarse en la práctica cotidiana del centro y de las aulas. El liderazgo ha de contemplar ineludiblemente las facetas curriculares y de enseñanza-aprendizaje, que son el núcleo del centro escolar.

Generar las condiciones y desarrollar prácticas que hagan posible que todos los alumnos puedan aprender en un contexto de igualdad, respeto y excelencia requiere una actuación desde el liderazgo, que propicie y apoye procesos de colaboración profesional entre docentes y entre éstos y los miembros de toda la comunidad escolar.

La constitución en los centros escolares de verdaderos equipos docentes, desde la base de un propósito y valores comunes, ha de permitir reflexionar y hablar sobre la práctica, para examinarla y depurarla con el objetivo de construir una cultura de colaboración en la que los docentes se apoyen unos a otros para ensayar nuevas formas de enseñanza. Como proponen Ainscow y Miles $(2008,29)$ :

El intercambio de experiencias permite que los colegas se ayuden unos a otros a expresar lo que están haciendo y a definir lo que les gustaría hacer. Es también la forma de crear un espacio en el que se puedan someter a crítica recíproca las premisas admitidas sobre determinados grupos de educandos.

Los equipos docentes han de desarrollar la capacidad de generar confianza, que les permitirá realizar un trabajo colaborativo efectivo y duradero para enfrentarse a los conflictos y a las cuestiones y dudas que surgen en la cotidianidad de los centros escolares. La constitución de redes internas de apoyo representa un elemento capital en la consolidación de las propuestas de prácticas inclusivas.

La implementación del modelo inclusivo en las instituciones escolares se evidencia en el modelo organizativo que se adopta, pues éste delimita las principales reglas y normas de funcionamiento, que favorecen o impiden la consolidación de la educación inclusiva. La organización, por tanto, es un instrumento, un medio, que ha de posibilitar un proceso 
de enseñanza-aprendizaje con unas características determinadas y una adecuada atención a la diversidad, con propuestas globales y previas, para propiciar unas condiciones necesarias de cara a la intervención satisfactoria en el aula (Torres, 2012)

Existen una serie de principios generales de organización escolar y práctica de aula que, siguiendo a Ainscow (2012) deberían respetarse: a) la eliminación de las barreras entre los distintos grupos de alumnos y el personal docente; b) el desmantelamiento de programas, servicios y especialidades aisladas; y c) el desarrollo de enfoques pedagógicos que permitan a los alumnos aprender juntos y no separados. Estos principios se concretan, a su vez, en dos decisiones organizativas relevantes y decisivas: por una parte, las formas de agrupamiento de los alumnos; por otra parte, la organización de los apoyos.

\section{Agrupamiento de LOS ALUMNOS}

Moran, Molina y Siles (2012), fruto del proyecto Includ-Ed, presentan tres modalidades de agrupación del alumnado, que implican formas diferentes de responder a la diversidad y que provocan resultados educativos encontrados:

a) Mixtures. Se trata de un modelo organizativo propio del aula tradicional, donde un único profesor atiende a un alumnado heterogéneo, que comparte unos mismos objetivos, contenidos y actividades de aprendizaje. En la práctica, el desarrollo de este modelo encuentra serias dificultades para dar respuesta a un alumnado diverso.

b) Streaming. Se separa al alumnado por niveles de rendimiento, en diferentes aulas o dentro de la misma, añadiendo más recursos humanos para atender a los diferentes grupos. Bajo esta organización son los estudiantes con más dificultades los que resultan más perjudicados en su aprendizaje, lo cual repercute tanto en la calidad como en la equidad educativa.

c) Inclusión. Las iniciativas referidas en este modelo se basan en mantener la heterogeneidad del aula, pero incorporando los recursos en el aula ordinaria, donde todos los alumnos comparten el mismo entorno y un mismo contenido de aprendizaje.

La propuesta inclusiva se fundamenta en optar siempre, y en todas las actividades y ocasiones, por agrupamientos heterogéneos, con lo que se 
evita la segregación y la discriminación del alumnado más vulnerable y se potencia la calidad y equidad de la propuesta educativa. Además, con este modelo es posible alcanzar un mayor nivel de colaboración entre los alumnos, lo cual aumenta la motivación y la oportunidad de ayuda mutua en el aprendizaje. Como destacan Puigdellivol y Krastina (2010) los agrupamientos heterogéneos con la reorganización de los apoyos existentes, muestran una incidencia positiva en el rendimiento de todos los alumnos y también favorecen el establecimiento de relaciones, al trabajar cooperativamente y al crear relaciones de ayuda y solidaridad entre el alumnado.

El proyecto Includ-Ed (Ballesteros, 2011) muestra en sus conclusiones que existe una correlación negativa entre agrupamientos homogéneos y los resultados académicos obtenidos; también muestra que siempre resultan más perjudicados los alumnos con peor rendimiento, lo cual es la causa principal de la generación de desigualdades entre el alumnado. En el otro extremo, aparecen los agrupamientos inclusivos, mediante los cuales el alumno más vulnerable puede ampliar sus oportunidades de aprendizaje, obtener mejores resultados, establecer relaciones sociales más positivas y mejorar su autoestima, sin perjudicar en ningún caso a los alumnos con más capacidades.

\section{ORGANIZACIÓN DE LOS APOYOS.}

Desde el enfoque inclusivo, se reclaman la necesaria incorporación de los recursos humanos al aula ordinaria, para trabajar conjuntamente con los tutores y favorecer la participación y el aprendizaje de todos los alumnos del grupo, en todas las experiencias y actividades planteadas. Así, las funciones del profesorado de apoyo son más de coordinación y de asesoramiento, que de atención directa al alumnado, lo que facilita la responsabilidad compartida. Como señalan Sandoval, Simón y Echeita (2012), el papel principal del apoyo es asesorar a los profesores de aula para utilizar técnicas, estrategias, alternativas y metodologías que favorezcan la inclusión de todos los alumnos y que aseguren mecanismos de coordinación y trabajo conjunto.

Muntaner (2009) propone una serie de actuaciones a desarrollar para la aplicación de un modelo de apoyo coherente con el modelo de escuela inclusiva, actuaciones que se concretan en los siguientes puntos:
A. Apoyo como recurso para atender a la diversidad.
B. Colaborar en la diversificación del proceso de aprendizaje. 
C. Potenciar la organización dinámica y flexible del aula.

D. El apoyo con la aportación de ideas, estrategias y alternativas didácticas al funcionamiento general de la clase.

E. Participar en el proceso de evaluación.

F. El apoyo potencia una autonomía del trabajo de los alumnos.

G. El aula es el lugar natural de participación del apoyo.

H. El apoyo ayuda a modificar las expectativas y actitudes del profesor.

La concreción de todas las propuestas anteriormente explicadas se plasma en el desarrollo de las prácticas en el aula. Crear un aula inclusiva es un reto, pues ha de ser un espacio de todos, desarrollado como una comunidad de participación para todos; ello exige introducir en el aula estrategias y prácticas diferentes de las utilizadas tradicionalmente.

\section{Una apuesta de futuro a modo de conclusión}

La inclusión no es una simple cuestión organizativa, curricular o metodológica; es una manera distinta de entender y practicar la educación, que propone y exige metodologías didácticas, propuestas curriculares y modelos organizativos donde puedan aprender juntos alumnos diferentes.

La educación inclusiva se fundamenta en tres principios claves: la calidad, la equidad y la inclusión. Aprender a trabajar en los centros escolares con la diversidad del alumnado, manteniendo la igualdad de oportunidades y las altas expectativas de éxito para todos los alumnos, representa los dos grandes retos del modelo educativo actual.

Las buenas prácticas inclusivas tienen dos características fundamentales: Primera, se trabaja siempre con grupos heterogéneos de alumnos, porque las categorías no aportan nada a nuestro trabajo. Segunda, se cuenta con apoyos, ya sean humanos o materiales, desarrollados siempre en el aula regular, en beneficio de la participación y del aprendizaje de cualquier alumno. Pensamos y defendemos que el problema no está en el alumno ni en sus características, sino en las oportunidades y en los medios que se ponen a su disposición para favorecer su pleno desarrollo.

La implantación y consolidación de buenas prácticas inclusivas requiere cambios sustanciales en la cultura de los centros y las aulas. Un cambio que afecta a las creencias, concepciones, ideas y principios del 
profesorado, ya que modifica sus referencias para interpretar la realidad. Esta interpretación de la realidad modifica los planteamientos curriculares, organizativos y metodológicos que se aplican, al contemplar la diversidad de los alumnos como un hecho natural con el que es posible trabajar. La puesta en práctica de metodologías abiertas, flexibles y comunes, que favorecen el éxito de todos los alumnos y luchan contra la exclusión es el resultado de este proceso.

El gran reto al que nos enfrentamos hoy está en la consolidación y generalización de estos ideales y de estas prácticas. Sabemos y somos conscientes que la educación inclusiva es el modelo educativo que mayor calidad y equidad puede ofrecernos para que todo el alumnado alcance el éxito en la escuela. Sabemos que el camino es largo, tortuoso, convulso y complejo, pero apostamos decididamente por alcanzar una educación sin exclusiones, porque estamos convencidos de la necesidad del cambio y somos valientes y firmes en nuestras creencias y en la defensa de estos ideales.

\section{Referencias bibliográficas}

Agell, M.; Sala, G. y Torrent, J. (2009). Participación de todo el alumnado, éxito y mejora de la escuela. Análisis de las barreras más relevantes y cómo superarlas. En Giné, C. (Coord), La educación inclusiva. (63-80). Barcelona: Horsori.

Agencia Europea para el Desarrollo de la Educación del Aumnado con Necesidades Educativas Especiales (2009). Principios fundamentales para la promoción de la calidad de la educación inclusiva. Recuperado de http://www.european-agency.org/ sites/default/files/key-principles-for-promoting-quality-in-inclusive-education_keyprinciples-ES.pdf

Ainscow, M. (2005a). Developing inclusive education systems: what are the levers for change. Journal of Educational Change, 6 (2). 109-124.

Ainscow, M. (2005b). El próximo gran reto: la mejora de la escuela inclusiva. (Presentación de apertura del Congreso sobre efectividad y Mejora Escolar. Barcelona).

Ainscow, M. (2012). Haciendo que las escuelas sean más inclusivas: lecciones a partir del análisis de la investigación internacional. Revista de Educación Inclusiva, 5 (1), 39-49.

Ainscow, M. y Miles, S. (2008). Por una educación para todos que sea inclusiva: ¿hacia dónde vamos ahora? Perspectivas, XXXVIII (1), 17-44.

Ainscow, M.; Booth, T. y Dyson (2006). Improving Schools, Developing Inclusion. Nueva York: Routledge.

Albericio, J.J. (2001). Las agrupaciones flexibles, reflexión crítica desde la experiencia. AAVV. Estrategias organizativas de aula (29-36). Barcelona: Graó. 
Arnaiz, P. (2011). Luchando contra la exclusión: buenas prácticas y éxito escolar. Innovación educativa, 21, 23-35.

Arnaiz, P. (2012). Escuelas eficaces e inclusivas cómo favorecer su desarrollo. Educatio Siglo XXI, 30 (1), 25-44.

Ballesteros, A. (2011). Actuaciones de éxito para superar la exclusión Educativa en Europa. Conferencia Final del proyecto INCLUD-ED. Bruselas, sede del Parlamento Europeo.

Blanco, R. (2008). Marco conceptual sobre educación inclusiva. 48 $8^{a}$ Reunión de la Conferencia Internacional de Educación. La educación inclusiva: el camino hacia el futuro. Documento Final. Recuperado de: http:// www.uam.es/personal_pdi/stmaria/ sarrio/declaraciones\%20manifiestos/ICE_FINAL_REPORT_spa\%5B1\%5D.pdf

Booth, T. (2006). Manteniendo el futuro con vida; convirtiendo los valores de inclusión en acciones. En M.A.Verdugo y B. Jordan de Urríes. (Coords.), Rompiendo inercias. Claves para avanzar VI Jornadas científicas de investigación sobre personas con discapacidad (211-218). Salamanca: Amarú.

Booth, T. y Ainscow, M. (2002). Guía para la evaluación y mejora de la educación inclusiva. Paris: UNESCO.

Casanova, M.A. (2011). Educación inclusiva: un modelo de futuro. Madrid: Wolters Kluwer.

Echeita, G. (2006). Educación para la inclusión o educación sin exclusiones. Madrid: Narcea.

Echeita, G. y Ainscow, M. (2011). La educación inclusiva como derecho. Marco de referencia y pautas de acción para el desarrollo de una revolución pendiente. Tejuelo, 12, 26-46.

Elboj, C., Puigdellivol, I., Soler, M. y Valls, R. (2002). Comunidades de aprendizaje. Transformar la educación. Barcelona: Graó.

Escudero, J.M. (2009). Buenas prácticas y programas extraordinarios de atención al alumnado en riesgo de exclusión educativa. Revista de currículum y formación del profesorado, 13 (3), 108-141.

Escudero, J.M. y Martínez, B. (2011). Educación inclusiva y cambio escolar. Revista Iberoamericana de educación, 55, 85-105.

Escudero, J.M. y Martínez, B. (2012). Las políticas de lucha contra el fracaso escolar: ¿programas especiales o cambio profundos del sistema y la educación? Revista de Educación, № Extraordinario,174-193.

Florian, L.; Black-Hawkins, K (2010). Exploring inclusive pedagogy. British Educational Research Journal, 32 (1), 1-16.

García, M. y otros (2012). Medidas eficaces en atención a la diversidad cultural desde una perspectiva inclusiva. Revista de Educación, 358, 258-281.

Giné, Cl. (Coord) (2010). La educación inclusiva. De la exclusión a la plena participación de todo el alumnado. Barcelona: ICE-Horsori.

González, M.T. (2008). Diversidad e inclusión educativa: algunas reflexiones sobre el liderazgo en el centro escolar. REICE. Revista Electrónica Iberoamericana sobre calidad, eficacia y cambio en educación, 6 (2), 82-99.

Halinen, I; Jarvinen, R. (2008). En pos de la educación inclusiva: el caso de Finlandia. Perspectivas, XXXVIII (1), 97-127. 
López Melero, M. (2004). Construyendo una escuela sin exclusiones: una forma de trabajar en el aula con proyectos de investigación. Málaga: Aljibe.

Marchesi, A. y otros (2009). Guía para la reflexión y valoración de prácticas inclusivas. Organización de estados iberoamericanos para la educación, la ciencia y la cultura. Madrid. www.oei.es/iclusivamapfre/guia.pdf

Martínez, A. Y Gómez, J.L. (coord.) (2013). Escuelas inclusivas singulares. Madrid: Grupo5.

Murillo, F.J. y Krichesky, G.J. (2012). El proceso de cambio escolar. Una guía para impulsar y sostener la mejora de las escuelas. Revista Iberoamericana sobre Calidad, Eficacia y Cambio en Educación, 10 (1), 26-43.

Moran, C.; Molina, S. y Siles, G. (2012). Aportaciones científicas a las formas de agrupación del alumnado. Revista de Organización y Gestión Educativa, 2, 13-18.

Muntaner, J. J. (2009). Escuela y discapacidad intelectual. Sevilla: Eduforma.

Muntaner, J. J. (2010). De la integración a la inclusión: un nuevo modelo educativo. En P. Arnaiz, M.D. Hurtado, y F.J.Soto, 25 años de integración escolar en España. Murcia: Consejeria de Educación, Formación y Empleo.

OCDE (2012). Equity and Quality in Education. Supporting Disadvantaged Students and Schools. OECD.

Parrilla, A. (2009). ¿Y si la investigación sobre inclusión no fuera inclusiva?. Reflexiones desde una investigación biografía-narrativa. Revista de Educación, 349, 101-118.

Puigdellivol, I.; Krastina, L. (2010). Inclusió i segregació a I'escola: pràctiques inclusives i excloents amb l'alumnat vulnerable. Temps d'Educació, 38, 95-113.

Sandoval, M.; Simón, C.: Echeita, G. (2012). Análisis y valoración crítica de las funciones del profesorado de apoyo desde una educación inclusiva. Revista de Educación, № Extraordinario, 117-137.

Santiso, C. (2002). Education for democratic governance: review of learning programmes. Unesco. Org. Social Sciences.

Torres, J.A. (2012). Estructuras organizativas para una escuela inclusiva: promoviendo comunidades de aprendizaje. Educatio Siglo XXI, 30 (1), 45-70.

Verdugo, M.A.; Parrilla, A. (2009). Presentación y aportaciones actuales a la educación inclusiva. Revista de Educación, 349, 15-22.

Wehmeyer, M.L. (2009). Autodeterminación y la tercera generación de prácticas de inclusión. Revista de Educación, 349, 45-67. 
